\title{
ON THE MINIMAL NUMBER OF TRANSLATED POINTS IN CONTACT LENS SPACES
}

\author{
SIMON ALLAIS
}

\begin{abstract}
In this article, we prove that every contactomorphism of any standard contact lens space of dimension $2 n-1$ that is contact-isotopic to identity has at least $2 n$ translated points. This sharp lower bound refines a result of GranjaKarshon-Pabiniak-Sandon and answers a conjecture of Sandon positively.
\end{abstract}

\section{INTRODUCTION}

Given a contact manifold $(V, \operatorname{ker} \alpha)$, a discriminant point of a contactomorphism $\varphi \in \operatorname{Cont}(V, \operatorname{ker} \alpha)$ is a point $p \in V$ such that $\varphi(p)=p$ and $\left(\varphi^{*} \alpha\right)_{p}=\alpha_{p}$ (it does not depend on the choice of contact form). Generically, contactomorphisms do not possess any discriminant points. A translated point of $\varphi$ with time-shift $\tau$ is a discriminant point of $\phi_{-\tau}^{\alpha} \circ \varphi$, where $\left(\phi_{t}^{\alpha}\right)$ denotes the Reeb flow of $\alpha$; it does depend on the choice of contact form $\alpha$. Contactomorphisms of a closed manifold $V$ that are $C^{1}$-close to identity have at least as many translated points as some smooth maps $V \rightarrow \mathbb{R}$ (see below).

Inspired by the Arnol'd conjecture, Sandon asked whether any contactomorphism contact-isotopic to identity $\varphi \in \operatorname{Cont}_{0}(V)$ has at least as many translated points as the minimal number of critical points a smooth map $V \rightarrow \mathbb{R}$ can have. This question was positively answered by Sandon for contact spheres $\mathbb{S}^{2 n-1}$ and real projective spaces $\mathbb{R} P^{2 n-1}$ endowed with the standard contact form

$$
\alpha:=\frac{1}{2 \pi} \sum_{j=1}^{n}\left(x_{j} \mathrm{~d} y_{j}-y_{j} \mathrm{~d} x_{j}\right),
$$

(the normalization is made so that the Reeb flow $\phi_{t}^{\alpha}: z \mapsto e^{2 i \pi t} z$ is 1-periodic) [11]. In the case of hypertight contact manifold (i.e. such that all Reeb orbits are noncontractible for some contact form supporting the contact structure), the question was positively answered for generic contactomorphisms contact-isotopic to identity by the work of Albers-Fuchs-Merry [1] completed by Meiwes-Naef [10]. The case of the standard lens spaces was partially answered by Granja-Karshon-PabiniakSandon [8]. A lens space $L_{k}^{2 n-1}$ with fundamental group $\mathbb{Z} / k \mathbb{Z}$ is the quotient of $\mathbb{S}^{2 n-1}$ under the free $\mathbb{Z} / k \mathbb{Z}$-action generated by

$$
\left(z_{1}, \ldots, z_{n}\right) \mapsto\left(e^{2 i \pi w_{1} / k} z_{1}, \ldots, e^{2 i \pi w_{n} / k} z_{n}\right),
$$

where $w_{1}, \ldots, w_{n} \in \mathbb{N}^{*}$ are positive integers prime to $k$. This definition does depend on the choice of integers $w_{1}, \ldots, w_{n}$ but not the results stated here so we will not

Date: March 30, 2021.

2020 Mathematics Subject Classification. 53D10, 58E05, 57R17.

Key words and phrases. Arnold conjecture, generating functions, Lusternik-Schnirelmann theory, Reeb dynamics, translated points of contactomorphisms. 
write them explicitly. The standard contact form (1) is invariant under these $\mathbb{Z} / k \mathbb{Z}$ actions, endowing the associated lens spaces with their standard contact structure. In this article, we give a positive answer to the conjecture of Sandon in the case of standard contact lens spaces.

In order to state the general result of the paper properly, let us fix a free $\mathbb{Z} / k \mathbb{Z}$ action (2) and let us denote by $\operatorname{Cont}_{0}^{\mathbb{Z} / k \mathbb{Z}}\left(\mathbb{S}^{2 n-1}\right)$ the set of $\mathbb{Z} / k \mathbb{Z}$-equivariant contactomorphisms of $\mathbb{S}^{2 n-1}$ that are isotopic to identity through $\mathbb{Z} / k \mathbb{Z}$-equivariant contactomorphisms. The set of time-shifts of a contactomorphism $\varphi \in \operatorname{Cont}_{0}\left(\mathbb{S}^{2 n-1}\right)$ will refer to the set of time-shifts of its translated points. Since the Reeb flow of (1) is 1-periodic, the set of time-shifts of $\varphi$ is invariant under integral translations so it can be seen as a subset of $\mathbb{R} / \mathbb{Z}$.

Theorem 1.1. Let us assume that $\varphi \in \operatorname{Cont}_{0}^{\mathbb{Z} / k \mathbb{Z}}\left(\mathbb{S}^{2 n-1}\right)$ has finitely many translated points (with the standard contact form), then the number of time-shifts of $\varphi$ in $\mathbb{R} / \mathbb{Z}$ is at least $2 n$.

More precise information about the structure of the set of translated points of $\varphi$ can be also recovered from the proof, see Remark 2.2. Theorem 1.1 implies that every contactomorphism $\varphi \in \operatorname{Cont}_{0}\left(L_{k}^{2 n-1}\right)$ has at least $2 n$ translated points. Indeed, one can lift $\varphi$ to $\tilde{\varphi} \in \operatorname{Cont}_{0}^{\mathbb{Z} / k \mathbb{Z}}\left(\mathbb{S}^{2 n-1}\right)$. Translated points of $\widetilde{\varphi}$ come in families of $\mathbb{Z} / k \mathbb{Z}$ orbits and translated points of a same orbit have a common time-shift $t \in \mathbb{R} / \mathbb{Z}$. Since distinct $\mathbb{Z} / k \mathbb{Z}$-orbits of translated points of $\widetilde{\varphi}$ projects to distinct translated points of $\varphi$, the conclusion follows.

Corollary 1.2. Every contactomorphism of $L_{k}^{2 n-1}$ contact-isotopic to identity has at least $2 n$ translated points.

More precisely, Theorem 1.1 implies that every contactomorphism $\varphi_{1}$ of $L_{k}^{2 n-1}$ contact-isotopic to identity through $\left(\varphi_{t}\right)$ has at least $2 n$ translated points $\left\{x_{j}\right\}$ so that the loops

$$
t \mapsto \begin{cases}\varphi_{2 t}\left(x_{j}\right), & t \in[0,1 / 2], \\ \phi_{(1-2 t) \tau_{j}}^{\alpha} \circ \varphi_{1}\left(x_{j}\right), & t \in[1 / 2,1],\end{cases}
$$

are contractible for some choice of respective time-shifts $\left\{\tau_{j}\right\}$. Moreover, if the set $\left\{x_{j}\right\}$ is finite, the cardinal of the projection of $\left\{\tau_{j}\right\}$ in $\mathbb{R} / \mathbb{Z}$ is at least $2 n$ (and more information about the structure of these sets can be recovered from the proof, see Remark 2.2).

This lower bound is optimal. Indeed, let $\operatorname{gr}(\varphi)$ be the graph of a contactomorphism $\varphi \in \mathrm{Cont}_{0}(V, \alpha)$

$$
\operatorname{gr}(\varphi):=\{(p, \varphi(p), g(p)) \mid p \in V\} \subset V \times V \times \mathbb{R},
$$

where $\varphi^{*} \alpha=e^{g} \alpha$. The graph of $\varphi$ is a Legendrian for the contact form $\alpha_{2}-e^{\theta} \alpha_{1}$ where $\alpha_{j}$ is the pull-back of $\alpha$ by the projection on the $j$-th factor and $\theta$ denotes the coordinate of the $\mathbb{R}$ factor. Discriminant points of $\varphi$ correspond to intersection of $\operatorname{gr}(\varphi)$ with $\operatorname{gr}(\mathrm{id})=\Delta \times 0$ where $\Delta \subset V \times V$ is the diagonal. If $\varphi$ is $C^{1}$-close to identity, then $\operatorname{gr}(\varphi)$ lies in a Weinstein neighborhood $W \simeq J^{1} \Delta$ of $\Delta \times 0$. Seen in $J^{1} \Delta$, the graph of $\varphi$ is the 1 -jet $j^{1} f$ of a smooth map $f: \Delta \rightarrow \mathbb{R}$ and translated points with small time-shift $t$ correspond to critical points of $f$ with critical value $t$, as stated. In the special case where all Reeb orbits of $(V, \alpha)$ are closed, one can shrink the Weinstein neighborhood $W$ so that the Reeb orbits of gr(id) correspond 
only to Reeb orbits of $\Delta \times 0 \subset J^{1} \Delta$ (whereas if the Reeb orbit $\gamma$ of some point $(x, x, 0) \in \operatorname{gr}(\mathrm{id})$ is not closed, the Poincaré recurrence theorem implies that $\gamma$ contains a sequence of $\left(x, y_{k}, 0\right)$ with $y_{k} \rightarrow x$ and $y_{k} \neq x$; so that every Weinstein neighborhood must contain some point in the same Reeb orbit as $(x, x, 0)$ but in a different Reeb orbit of the neighborhood $\left.W \simeq J^{1} \Delta\right)$. Therefore, when all Reeb orbits of $(V, \alpha)$ are closed, there exists contactomorphisms contact-isotopic to identity with exactly as many translated points as the minimal number of critical point a map $V \rightarrow \mathbb{R}$ can have (this discussion was already present in the work of Sandon [11]).

Since the Reeb flow of $L_{k}^{2 n-1}$ is periodic, it is enough to find a map $L_{k}^{2 n-1} \rightarrow \mathbb{R}$ with $2 n$ critical points. A classical way of doing so is to consider the quotient map of the $\mathbb{Z} / k \mathbb{Z}$-invariant map of $\mathbb{S}^{2 n-1}$

$$
\left(z_{1}, \ldots, z_{n}\right) \mapsto\left|z_{1}\right|^{2}+2\left|z_{2}\right|^{2}+\cdots+n\left|z_{n}\right|^{2} .
$$

This is a Morse-Bott map $L_{k}^{2 n-1} \rightarrow \mathbb{R}$ whose critical set is the disjoint union of $n$ circles. By properly adding a Morse map of the circle with 2 critical points in the Morse-Bott neighborhood of each critical circle, one obtains a Morse map with $2 n$ critical points.

Since every $\mathbb{Z} / k \mathbb{Z}$-equivariant map of $\mathbb{S}^{2 n-1}$ is also equivariant under a subgroup $\mathbb{Z} / p \mathbb{Z} \subset \mathbb{Z} / k \mathbb{Z}$ where $p$ is a prime dividing $k$, it is enough to prove Theorem [1.1] when $k$ is a prime. The case when $k$ is even was already proven by Sandon [11]. Granja-Karshon-Pabiniak-Sandon proved Theorem 1.1 when translated points of $\varphi$ are non-degenerate and proved that the number of translated points is at least $n$ otherwise [8, Corollary 1.3].

In order to study translated points, we follow the idea of Sandon originally used in the case of $\mathbb{S}^{2 n-1}$ and $\mathbb{R P}^{2 n-1}$ in [11] and extended by Granja-Karshon-PabiniakSandon to lens spaces $L_{k}^{2 n-1}$ in [8]. Sandon discovered that the proofs of the Arnol'd conjecture in $\mathbb{C P}^{n-1}$ given by Givental [7] and Théret [12] can be extended to study translated points on quotients of the contact sphere $\mathbb{S}^{2 n-1}$. In this article we are deeply inspired by the point of view of Théret on the Fortune-Weinstein theorem [6] that we had already develop to study periodic points of Hamiltonian diffeomorphisms in $\mathbb{C P}^{n-1}$ [2, 3] whereas the study of Granja-Karshon-Pabiniak-Sandon was mainly focused on the properties of a quasimorphism inspired by Givental's nonlinear Maslov index on $\mathbb{C P}^{n-1}$.

In order to study the $\mathbb{Z} / k \mathbb{Z}$-equivariant contactomorphism $\varphi \in \operatorname{Cont}_{0}^{\mathbb{Z} / k \mathbb{Z}}\left(\mathbb{S}^{2 n-1}\right)$ of the sphere $\mathbb{S}^{2 n-1} \subset \mathbb{C}^{n}$, we lift it to a Hamiltonian diffeomorphism $\Phi$ of the symplectization $\mathbb{C}^{n} \backslash 0$ (endowed with the standard symplectic form $\mathrm{d} x \wedge \mathrm{d} y$ )

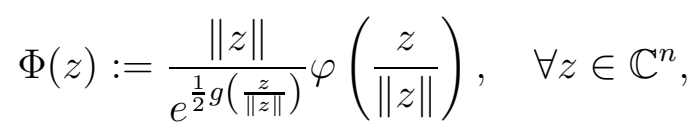

where $\varphi^{*} \alpha=e^{g} \alpha$ and $\|\cdot\|$ denotes the usual Euclidean norm. By construction, $\Phi$ is $\mathbb{R}_{+}$-homogeneous and equivariant under the $\mathbb{Z} / k \mathbb{Z}$-action extended by linearity from the action on $\mathbb{S}^{2 n-1}: \Phi$ is an $\left(\mathbb{R}_{+} \times \mathbb{Z} / k \mathbb{Z}\right)$-equivariant Hamiltonian diffeomorphism. $\mathbb{Z} / k \mathbb{Z}$-orbits of translated points with time-shift $t$ then correspond to $\left(\mathbb{R}_{+} \times \mathbb{Z} / k \mathbb{Z}\right)$-orbits of fixed points of $e^{-2 i \pi t} \Phi$. For such families of Hamiltonian diffeomorphisms $\left(e^{-2 i \pi t} \Phi\right)$, Givental and Théret developed a variational theory based on generating functions (they restricted themselves to homogeneous Hamiltonians 
invariant under $S^{1}$ or $\mathbb{Z} / 2 \mathbb{Z}$, the extension to $\mathbb{Z} / k \mathbb{Z}$ was considered by GranjaKarshon-Pabiniak-Sandon). Theorem 1.1 follows from a careful application of the Lusternik-Schnirelmann theory to this variational setting.

Organization of the paper. In Section 2, we recall results of the LusternikSchnirelmann theory that are useful for us. In Section 3, we introduce the variational setting and use it to prove Theorem 1.1.

Acknowledgment. I am grateful to Yael Karshon and Margherita Sandon for their advices and support. I also thank Daniel Álvarez-Gavela, Mohammed Abouzaid and the other organizers of the Generating Function Day of February 2021, where I was able to discuss this subject with Yael for the first time. Finally, I would like to thank my advisor Marco Mazzucchelli for his constant support and helpful advice.

\section{LusterniK-SCHNiRELMANN THEORY ON THE RELATIVE CASE}

2.1. Lusternik-Schnirelmann theorem. Let $X$ be an ANR topological space (metric is enough for us). Given $A \subset X$, the integer cat $X A \in \mathbb{N}$ is the minimal integer $k$ such that $A$ can be covered by $k$ open subsets that are contractible in $X$. The Lusternik-Schnirelmann category of $X$ is defined as

$$
\text { cat } X:=\operatorname{cat}_{X} X \text {. }
$$

Let us fix $Y \subset X$. Given a subset $A \subset X$ that contains $Y$, the integer $\operatorname{cat}_{X}(A, Y) \in$ $\mathbb{N}$ is the minimal integer $k$ such that

$$
A \subset W \cup A_{1} \cup A_{2} \cup \cdots \cup A_{k},
$$

with $W \subset X$ open that contains $Y$ and retracts on $Y$ by deformation and open subsets $A_{1}, \ldots, A_{k}$ that are contractible in $X$. The Lusternik-Schnirelmann category of $(X, Y)$ is defined as

$$
\operatorname{cat}(X, Y):=\operatorname{cat}_{X}(X, Y) .
$$

Let $f: M \rightarrow \mathbb{R}$ be a $C^{1}$-map defined on a closed manifold $M$. More generally, the following theorem is true for $f$ defined on a Banach manifold $M$ and that satisfies the Palais-Smale condition in $f^{-1}(a, b)$.

Theorem 2.1 (See for instance [4, Proposition 7.7]). Let $a \leq b$ be regular values of $f$. Let us assume that $\{f \leq b\}$ is connected. If $f$ has finitely many critical points with value inside $(a, b)$, the number of critical values $N \in \mathbb{N}$ inside $(a, b)$ satisfies

$$
N \geq \operatorname{cat}(\{f \leq b\},\{f \leq a\}) .
$$

Remark 2.2. Actually, the Lusternik-Schnirelmann theorem tells us a bit more about the structure of the critical set $K \subset f^{-1}(a, b)$ of $f$. For instance, if $f$ has less critical values than the category of its relative sublevel set, non only is the set of critical points $K$ infinite, but any neighborhood $V \subset\{f \leq b\}$ of $K$ has a category cat $_{X} V \geq 1$ relative to $X:=\{f \leq b\}$ (in particular, $V$ possess a non-contractible connected component). The proof of Theorem 1.1 is an application of this theorem and therefore tells us a bit more than stated too. In particular, if $\varphi \in \operatorname{Cont}_{0}^{\mathbb{Z} / k \mathbb{Z}}\left(\mathbb{S}^{2 n-1}\right)$ has less time-shifts in $\mathbb{R} / \mathbb{Z}$ than $2 n$, for any $\mathbb{Z} / k \mathbb{Z}$-invariant neighborhood $V \subset \mathbb{S}^{2 n-1}$, the inclusion $V /(\mathbb{Z} / k \mathbb{Z}) \hookrightarrow L_{k}^{2 n-1}$ is not null-homotopic. 
2.2. Fadell-Husseini's category weight. In the study of critical points of the lens spaces, the usual bound cat $X \geq C L(X)+1$, where $C L(X) \in \mathbb{N}$ denotes the cup-length of $X$, is not optimal. Given $u \in H^{*}(X)$ and $A \subset X$, the class $\left.u\right|_{A} \in H^{*}(A)$ denotes the image of $u$ under the restriction morphism induced by the inclusion $A \subset X$. Given $u \in H^{*}(X, Y)$ and $(A, B) \subset(X, Y)$, we denote by $\left.u\right|_{(A, B)} \in H^{*}(A, B)$ the restricted class.

Given a non-zero class $u \in H^{*}(X)$, Fadell-Husseini [5] defined the category weight $\operatorname{cwgt}(u)$ of $u$ as the maximal integer $k \in \mathbb{N}$ such that $\left.u\right|_{A}=0$ for all $A \subset X$ satisfying cat $_{X} A \leq k$. It can be naturally extended to non-zero relative classes $u \in H^{*}(X, Y)$ as the maximal integer $k \in \mathbb{N}$ such that $\left.u\right|_{(A, Y)}=0$ for all $A \supset Y$ in $X$ satisfying $\operatorname{cat}_{X}(A, Y) \leq k$. For instance, if $X$ is connected, the class $1 \in H^{0}(X)$ has category weight $\operatorname{cwgt}(1)=0$ whereas $\operatorname{cwgt}(u) \geq 1$ for a non-zero class $u \in H^{d}(X)$ with degree $d \geq 1$. In the case of a non-zero relative class $u \in H^{*}(X, Y)$, the category weight may vanish.

By the usual properties of the cup-product, for non-zero classes $u$ and $v$ in $H^{*}(X)$ or $H^{*}(X, Y)$, if $u \smile v \neq 0$, then

$$
\operatorname{cwgt}(u \smile v) \geq \operatorname{cwgt}(u)+\operatorname{cwgt}(v) .
$$

Indeed, if $\left.u\right|_{A}=0$ and $\left.v\right|_{B}=0$ then $\left.(u \smile v)\right|_{A \cup B}=0$.

By definition of the category weight, $\operatorname{cat}(X, Y) \geq 1+\operatorname{cwgt}(u)$ for all non-zero class $u \in H^{*}(X, Y)$. Therefore, if $u_{1} \smile \cdots \smile u_{k}$ is a non-zero class of $H^{*}(X, Y)$, then

$$
\operatorname{cat}(X, Y) \geq 1+\operatorname{cwgt}\left(u_{1}\right)+\cdots+\operatorname{cwgt}\left(u_{k}\right) .
$$

In principle, it generalises the cup-length estimate. The following theorem shows that it is indeed a better estimate in general. Let us denote the mod $p$ Bockstein morphism of a topological space $X$ by $\mathcal{B}: H^{*}\left(X ; \mathbb{F}_{p}\right) \rightarrow H^{*+1}\left(X ; \mathbb{F}_{p}\right)$.

Theorem 2.3 ([5, Theorem 1.2]). Let $X$ be an ANR topological space and $p$ be a prime number. If $u \in H^{1}\left(X ; \mathbb{F}_{p}\right)$ and $\mathcal{B} u \neq 0$, then $\operatorname{cwgt}(\mathcal{B} u) \geq 2$.

As an application, one can compute the Lusternik-Schnirelmann category of a lens space $L_{p}^{2 n-1}$ of dimension $2 n-1$ and fundamental group $\mathbb{Z} / p \mathbb{Z}$. As a graded $\mathbb{F}_{p}$-algebra,

$$
H^{*}\left(L_{p}^{2 n-1} ; \mathbb{F}_{p}\right) \simeq \mathbb{F}_{p}[\alpha, \beta] /(\alpha)\left(\beta^{n}\right),
$$

where $\operatorname{deg} \alpha=1, \operatorname{deg} \beta=2$ and $\beta=\mathcal{B} \alpha$. The category weight $\operatorname{cwgt}(\alpha) \geq 1$ whereas $\operatorname{cwgt}(\beta) \geq 2$ according to Theorem 2.3 . Therefore

$$
\operatorname{cat}\left(L_{p}^{2 n-1}\right) \geq 1+\operatorname{cwgt}\left(\alpha \smile \beta^{n-1}\right) \geq 1+\operatorname{cwgt}(\alpha)+(n-1) \operatorname{cwgt}(\beta) \geq 2 n .
$$

As there exist smooth maps $L_{p}^{2 n-1} \rightarrow \mathbb{R}$ with exactly $2 n$ critical points, Theorem 2.1 implies that $\operatorname{cat}\left(L_{p}^{2 n-1}\right)=2 n$. More precisely, Theorem 2.1 implies that every smooth map $L_{p}^{2 n-1} \rightarrow \mathbb{R}$ with finitely many critical points has at least $2 n$ critical values. The computation of $\operatorname{cat}\left(L_{p}^{2 n-1}\right)$ is initially due to Krasnosel'skiu [9].

\section{The optimal Bound ON TRANSLATED POINTS IN LENS SPACES}

In Section 3.1, we recall the variational principle for translated points. As we said in the introduction, it goes back to Givental [7] and Théret [12] to study Hamiltonian dynamics of $\mathbb{C P}^{n-1}$ and was then extended by Sandon [11] and Granja-KarshonPabiniak-Sandon [8] to treat translated points of quotients of $\mathbb{S}^{2 n-1}$. We do not 
strictly follow the lines of [8] but rather the point of view developed in [2, 3], inspired by ideas of Théret.

3.1. A variational principle for translated points. Let $p$ be a prime number greater than 2 and let us fix $\varphi \in \operatorname{Cont}_{0}^{\mathbb{Z} / p \mathbb{Z}}\left(\mathbb{S}^{2 n-1}\right)$. Let $\Phi \in \operatorname{Ham}\left(\mathbb{C}^{n} \backslash 0\right)$ be the $\left(\mathbb{R}_{+} \times\right.$ $\mathbb{Z} / p \mathbb{Z}$ )-equivariant lift given by (3). Let us build a $C^{1}$-family of generating functions $F_{t}:\left(\mathbb{C}^{2 n}\right)^{k} \rightarrow \mathbb{R}, t \in(-1,3)$, associated with $e^{-2 i \pi t} \Phi$ that is 2-homogeneous and invariant under the diagonal action of $\mathbb{Z} / p \mathbb{Z}$ on $\left(\mathbb{C}^{2 n}\right)^{k}$ induced by the action on $\mathbb{C}^{2 n}$. Let $\sigma_{1}, \ldots, \sigma_{m}$ be $\left(\mathbb{R}_{+} \times \mathbb{Z} / p \mathbb{Z}\right)$-equivariant Hamiltonian diffeomorphisms of $\mathbb{C}^{n} \backslash 0$ whose restriction to $\mathbb{S}^{2 n-1}$ are $C^{1}$-close to the identity such that $\Phi=\sigma_{m} \circ \cdots \circ \sigma_{2} \circ \sigma_{1}$, with $m$ even. Since the $\sigma_{j}$ 's are $C^{1}$-close to identity among $\left(\mathbb{R}_{+} \times \mathbb{Z} / p \mathbb{Z}\right)$-equivariant maps, there exist smooth maps $f_{j}: \mathbb{C}^{n} \backslash 0 \rightarrow \mathbb{R}$ such that

$$
\forall z_{j} \in \mathbb{C}^{n}, \exists ! w_{j} \in \mathbb{C}^{n}, \quad w_{j}=\frac{z_{j}+\sigma_{j}\left(z_{j}\right)}{2} \quad \text { and } \quad \nabla f_{j}\left(w_{j}\right)=i\left(z_{j}-\sigma_{j}\left(z_{j}\right)\right),
$$

(the maps $G_{j}:=\nabla f_{j}$ can be found by the implicit function theorem and these $G_{j}$ are gradients because their graphs are Lagrangians). By equivariance of the $\sigma_{j}$ 's, one can choose the $f_{j}$ 's to be 2 -homogeneous and $\mathbb{Z} / p \mathbb{Z}$-invariant. These maps extend to $C^{1,1}$-maps of $\mathbb{C}^{n}$ by imposing $f_{j}(0)=0$. In the special case of the small equivariant Hamiltonian diffeomorphism $\delta_{t}: z \mapsto e^{-2 i \pi t} z$, the associated map $q_{t}: \mathbb{C}^{n} \rightarrow \mathbb{R}$ is

$$
q_{t}(w):=-\tan (\pi t)\|w\|^{2}, \quad \forall w \in \mathbb{C}^{n},
$$

well defined for $t \in(-1 / 2,1 / 2)\left(\|\cdot\|\right.$ denotes the usual Euclidean norm of $\left.\mathbb{C}^{n} \simeq \mathbb{R}^{2 n}\right)$. We can now define, for $v_{1}, \ldots, v_{m+7} \in \mathbb{C}^{n}$ and $t \in(-1,3)$,

$$
F_{t}\left(v_{1}, \ldots, v_{m+7}\right):=\sum_{j=1}^{m} f_{j}\left(\frac{v_{j}+v_{j+1}}{2}\right)+\sum_{j=m+1}^{m+7} q_{t / 7}\left(\frac{v_{j}+v_{j+1}}{2}\right)+\sum_{j=1}^{m+7} \frac{1}{2}\left\langle v_{j}, i v_{j+1}\right\rangle .
$$

Therefore, the map $(t, \mathbf{v}) \mapsto F_{t}(\mathbf{v})$ is a $C^{1}$-map $(-1,3) \times\left(\mathbb{C}^{n}\right)^{m+7} \rightarrow \mathbb{R}$ that is 2-homogeneous and $\left(\mathbb{R}_{+} \times \mathbb{Z} / p \mathbb{Z}\right)$-equivariant.

Proposition 3.1 ([2, Proposition 5.1]). Critical points $\left(v_{1}, \ldots, v_{m+7}\right)$ of $F_{t}$ are in one-to-one correspondence with fixed points of $e^{-2 i \pi t} \Phi$ through the map

$$
\left(v_{1}, \ldots, v_{m+7}\right) \mapsto v_{1} .
$$

Since $F_{t}$ is 2 -homogeneous and $\mathbb{Z} / p \mathbb{Z}$-invariant, an $\left(\mathbb{R}_{+} \times \mathbb{Z} / p \mathbb{Z}\right)$-orbit of fixed points of $e^{-2 i \pi t} \Phi$ corresponds to an $\left(\mathbb{R}_{+} \times \mathbb{Z} / p \mathbb{Z}\right)$-orbit of critical points of $F_{t}$ with critical value 0 . Let $L_{p}^{2 N-1}$ be the lens space of dimension $2 N-1$ with $N:=n(m+7)$ obtained by quotient of $\mathbb{S}^{2 N-1}$ under the free diagonal action of $\mathbb{Z} / p \mathbb{Z}$. Let $\widehat{F}_{t}$ be the $C^{1}$-map $L_{p}^{2 N-1} \rightarrow \mathbb{R}$ induced by the $\mathbb{Z} / p \mathbb{Z}$-invariant map $\left.F_{t}\right|_{\mathbb{S}^{2 N-1}}$. Now, an $\left(\mathbb{R}_{+} \times \mathbb{Z} / p \mathbb{Z}\right)$-orbit of fixed points of $e^{-2 i \pi t} \Phi$ corresponds to a critical point of $\widehat{F}_{t}$ with critical value 0 and this correspondence is a bijection.

Going back to our initial problem, $\mathbb{Z} / p \mathbb{Z}$-orbits of translated points of $\varphi$ with time-shift $t \in(-1,3)$ are in one-to-one correspondence with $\left(\mathbb{R}_{+} \times \mathbb{Z} / p \mathbb{Z}\right)$-orbits of fixed points of $e^{-2 i \pi t} \Phi$. Therefore, the $\mathbb{Z} / p \mathbb{Z}$-orbits of translated points of $\varphi$ with time-shift $t \in(-1,3)$ are in one-to-one correspondence with critical points of $\widehat{F}_{t}$ with critical value 0 . 
According to [2, Lemma 5.6], the $C^{1}$-map $(t, z) \mapsto \widehat{F}_{t}(z)$ is a submersion. Let $M \subset(-1,3) \times L_{p}^{2 N-1}$ be the $C^{1}$-submanifold

$$
M:=\left\{(t, z) \mid \widehat{F}_{t}(z)=0\right\}
$$

and $\mathcal{T}: M \rightarrow(-1,3)$ be the $C^{1}$-map induced by the projection on the first factor. The $\mathbb{Z} / p \mathbb{Z}$-orbits of translated points of $\varphi$ with time-shift $t \in(-1,3)$ are in oneto-one correspondence with critical points of $\mathcal{T}$ with critical value $t$. By definition (41), $\widehat{F}_{t}$ is decreasing with $t$ so $\left\{\widehat{F}_{t} \leq 0\right\}$ is increasing. We recall that we have an isomorphism of graded $H^{*}\left(L_{p}^{2 N-1}\right)$-algebra

$$
H^{*}(\{\mathcal{T} \leq b\},\{\mathcal{T} \leq a\}) \simeq H^{*}\left(\left\{\widehat{F}_{b} \leq 0\right\},\left\{\widehat{F}_{a} \leq 0\right\}\right)
$$

for $a \leq b$, where $H^{*}\left(L_{p}^{2 N-1}\right) \simeq H^{*}\left((-1,3) \times L_{p}^{2 N-1}\right)$ acts by cup-product. This isomorphism being induced by a continuous map, it also commutes with the Bockstein morphism $\mathcal{B}$. See for instance [2, Section 5.4].

Given $A \subset L_{p}^{2 N-1}$, the cohomological index of $A$ is defined as the rank of the induced restriction morphism in cohomology

$$
\text { ind } A:=\operatorname{rank}\left(H^{*}\left(L_{p}^{2 N-1} ; \mathbb{F}_{p}\right) \rightarrow H^{*}\left(A ; \mathbb{F}_{p}\right)\right) \in\{0,1, \ldots, 2 N\} \text {. }
$$

Equivalently, ind $A$ is the minimal $k \in \mathbb{N}$ such that the morphism $H^{k}\left(L_{p}^{2 N-1} ; \mathbb{F}_{p}\right) \rightarrow$ $H^{k}\left(A ; \mathbb{F}_{p}\right)$ is trivial. Our definition slightly differs from [8] since we are using singular cohomology instead of Čech cohomology: in general it could be less than their index but it coincides on open sets. The following lemma is proven in [8, §Contact Arnold conjecture]. We explain how to adapt the proof to our setting

Lemma 3.2. Let us assume that $\varphi$ does not have discriminant points, then

$$
\text { ind }\left\{\widehat{F}_{2} \leq 0\right\}-\text { ind }\left\{\widehat{F}_{0} \leq 0\right\}=4 n \text {. }
$$

More generally the variation of index between the sublevel at time $t$ and time $t+k$ is $2 n k$ for $k \in \mathbb{N}$ as long as everything is defined and $t$ is not a time-shift. Here, we are considering a variation between time 2 and 0 instead of considering it between time 1 and 0 due to a technical issue in the proof of Theorem 1.1.

Proof. Since $\varphi$ does not have translated points with time-shift $0, \widehat{F}_{0}$ and $\widehat{F}_{2}$ are submersion. Therefore, $\left\{\widehat{F}_{0} \leq 0\right\}$ and $\left\{\widehat{F}_{2} \leq 0\right\}$ are submanifolds with boundary. Taking collar neighborhoods, we deduce that their singular cohomologies are naturally isomorphic to their Čech cohomologies. According to [2, Proposition 5.2 and Lemma 5.5], one can find $\mathbb{Z} / p \mathbb{Z}$-equivariant automorphisms $A_{0}$ and $A_{2}$ of $\mathbb{C}^{N}$ such that $F_{j} \circ A_{j}=G \oplus Q_{j}$ where $Q_{j}$ are non-degenerated quadratic form of $\left(\mathbb{C}^{n}\right)^{7}$ that are invariant under the diagonal action of $\mathbb{Z} / p \mathbb{Z}$ and satisfy

$$
i\left(Q_{2}\right)-i\left(Q_{0}\right)=4 n,
$$

where $i(Q) \in \mathbb{N}$ denotes the index of the quadratic form $Q$ (the referred proofs do not discuss possible $\mathbb{Z} / p \mathbb{Z}$-symmetries but it directly follows from the explicit formulas of $A_{j}$ and $Q_{j}$ that are given). Therefore, the $\left\{\widehat{Q}_{j} \leq 0\right\}$ 's retract on lens spaces $\simeq L_{p}^{i\left(Q_{j}\right)-1}$ by deformation. The conclusion is now a direct consequence of $[8$, Proposition 3.9 (v) and Proposition 3.14]. 


\subsection{Proof of the main theorem.}

Proof of Theorem 1.1. As was already mentioned in the introduction, by taking a subgroup, one can assume that $\varphi \in \operatorname{Cont}_{0}^{\mathbb{Z} / p \mathbb{Z}}\left(\mathbb{S}^{2 n-1}\right)$ with $p$ prime. The case $p=2$ was already treated by Sandon [11] and can easily be recovered with small modifications of the following proof. Hence, we assume that $p \geq 3$ and take back notation of the latter section.

Without loss of generality, we assume that $\varphi$ does not have any discriminant point (i.e. translated point with time-shift 0 ). Let $k \in \mathbb{N}$ be the number of time-shifts of $\varphi$ seen in $\mathbb{R} / \mathbb{Z}$. The number of time-shifts in $[0,2]$ is thus $2 k$. According to the correspondence with critical values of $\mathcal{T}$, the number of critical values in $[0,2]$ is also $2 k$. If $\varphi$ has finitely many translated points, $\mathcal{T}$ has finitely many critical points. According to Theorem 2.1, if $\varphi$ has finitely many translated points,

$$
2 k \geq \operatorname{cat}\left(\left\{\left.\mathcal{T}\right|_{C} \leq 2\right\},\left\{\left.\mathcal{T}\right|_{C} \leq 0\right\}\right),
$$

for all connected component $C$ of $\{\mathcal{T} \leq 2\}$.

In order to conclude, let us show that this category is at least $4 n-1$ for a good choice of $C$. As a graded $\mathbb{F}_{p}$-algebra,

$$
H^{*}\left(L_{p}^{2 N-1} ; \mathbb{F}_{p}\right) \simeq \mathbb{F}_{p}[\alpha, \beta] /(\alpha)\left(\beta^{N}\right),
$$

where $\operatorname{deg} \alpha=1, \operatorname{deg} \beta=2$ and $\beta=\mathcal{B} \alpha$. According to Lemma 3.2, we have the following variation of index

$$
\text { ind }\left\{\widehat{F}_{2} \leq 0\right\}-\text { ind }\left\{\widehat{F}_{0} \leq 0\right\}=4 n \text {. }
$$

If ind $\left\{\widehat{F}_{0} \leq 0\right\}$ is even $=2 r$, then the classes

$$
\beta^{r}, \alpha \beta^{r}, \ldots, \beta^{r+2 n-1}, \alpha \beta^{r+2 n-1} \in H^{*}\left(L_{p}^{2 N-1} ; \mathbb{F}_{p}\right)
$$

are in the kernel of the restriction to $\left\{\widehat{F}_{0} \leq 0\right\}$ but not in the one of the restriction to $\left\{\widehat{F}_{2} \leq 0\right\}$. Therefore, there is a class $u \in H^{2 r}\left(\left\{\widehat{F}_{2} \leq 0\right\},\left\{\widehat{F}_{0} \leq 0\right\}\right)$ (associated with $\beta^{r}$ ) such that the class

$$
u \smile \alpha \beta^{2 n-1} \in H^{2 r+4 n-1}\left(\left\{\widehat{F}_{2} \leq 0\right\},\left\{\widehat{F}_{0} \leq 0\right\}\right)
$$

is non-zero. Hence,

$$
\operatorname{cwgt}\left(u \smile \alpha \beta^{2 n}\right) \geq \operatorname{cwgt}(\alpha)+(2 n-1) \operatorname{cwgt}(\beta) \geq 4 n-1,
$$

according to Theorem 2.3 (we recall that $\beta=\mathcal{B} \alpha$ ). The isomorphism of $H^{*}\left(L_{p}^{2 N-1}\right.$ )algebra commuting with the Bockstein morphism $\mathcal{B}$ (5) allows us to conclude that

$$
\operatorname{cat}\left(\left\{\left.\mathcal{T}\right|_{C} \leq 2\right\},\left\{\left.\mathcal{T}\right|_{C} \leq 0\right\}\right) \geq 1+\operatorname{cwgt}\left(u^{\prime} \smile \alpha \beta^{2 n}\right) \geq 4 n,
$$

where $u^{\prime}$ is the image of $u$ under the isomorphism (15) and $C$ is a connected component on which $u^{\prime} \smile \alpha \beta^{2 n}$ is non-zero (we recall that the cohomology of a disjoint union of sets is the direct sum of the cohomologies of these sets).

If ind $\left\{\widehat{F}_{0} \leq 0\right\}$ is odd $=2 r+1$, then the classes

$$
\alpha \beta^{r}, \beta^{r+1}, \ldots, \alpha \beta^{r+2 n-1}, \beta^{r+2 n} \in H^{*}\left(L_{p}^{2 N-1} ; \mathbb{F}_{p}\right)
$$

are in the kernel of the restriction to $\left\{\widehat{F}_{0} \leq 0\right\}$ but not in the one of the restriction to $\left\{\widehat{F}_{2} \leq 0\right\}$. Therefore, there is a class $u^{\prime} \in H^{2 r+1}(\{\mathcal{T} \leq 2\},\{\mathcal{T} \leq 0\})$ (associated with $\alpha \beta^{r}$ ) such that the class

$$
u^{\prime} \smile \beta^{2 n-1} \in H^{2 r+4 n-1}(\{\mathcal{T} \leq 2\},\{\mathcal{T} \leq 0\})
$$


is non-zero. Hence,

$$
\operatorname{cat}\left(\left\{\left.\mathcal{T}\right|_{C} \leq 2\right\},\left\{\left.\mathcal{T}\right|_{C} \leq 0\right\}\right) \geq 1+\operatorname{cwgt}\left(u^{\prime} \smile \beta^{2 n-1}\right) \geq 1+2(2 n-1) \geq 4 n-1,
$$

where $C$ is a connected component on which $u^{\prime} \smile \beta^{2 n-1}$ is non-zero.

\section{REFERENCES}

1. Peter Albers, Urs Fuchs, and Will J. Merry, Orderability and the Weinstein conjecture, Compos. Math. 151 (2015), no. 12, 2251-2272.

2. Simon Allais, On periodic points of Hamiltonian diffeomorphisms of $\mathbb{C P}^{d}$ via generating functions, arXiv e-prints (2020), arXiv:2004.02165.

3. by Egor Shelukhin), arXiv e-prints (2020), arXiv:2010.14172.

4. Octav Cornea, Gregory Lupton, John Oprea, and Daniel Tanré, Lusternik-Schnirelmann category, vol. 103, Providence, RI: American Mathematical Society (AMS), 2003 (English).

5. Edward Fadell and Sufian Husseini, Category weight and Steenrod operations, Bol. Soc. Mat. Mexicana (2) 37 (1992), no. 1-2, 151-161, Papers in honor of José Adem.

6. Barry Fortune, A symplectic fixed point theorem for $\mathbf{C P}^{n}$, Invent. Math. 81 (1985), no. 1, 29-46.

7. Alexander B. Givental', Nonlinear generalization of the Maslov index, Theory of singularities and its applications, Adv. Soviet Math., vol. 1, Amer. Math. Soc., Providence, RI, 1990, pp. $71-103$.

8. Gustavo Granja, Yael Karshon, Milena Pabiniak, and Sheila Sandon, Givental's non-linear Maslov index on lens spaces, arXiv e-prints (2017), arXiv:1704.05827.

9. Mark A. Krasnosel' skiı̌, On special coverings of a finite-dimensional sphere, Dokl. Akad. Nauk SSSR (N.S.) 103 (1955), 961-964.

10. Matthias Meiwes and Kathrin Naef, Translated points on hypertight contact manifolds, J. Topol. Anal. 10 (2018), no. 2, 289-322.

11. Sheila Sandon, A Morse estimate for translated points of contactomorphisms of spheres and projective spaces, Geom. Dedicata 165 (2013), 95-110.

12. David Théret, Rotation numbers of Hamiltonian isotopies in complex projective spaces, Duke Math. J. 94 (1998), no. 1, 13-27.

Simon Allais, École Normale Supérieure de Lyon, UMPA

46 allée d'Italie, 69364 Lyon Cedex 07, France

Email address: simon.allais@ens-lyon.fr

$U R L$ : http://perso.ens-lyon.fr/simon.allais/ 